\title{
EVALUACIÓN DE CINCO PARÁMETROS DE CALIDAD EN FRUTA DE LA MORA DE CASTILLA Rubus glaucus BENTH VARIEDAD SIN ESPINAS COMPARADA CON LA VARIEDAD CON ESPINAS, EN CULTIVOS DE LA ZONA SUR DEL DEPARTAMENTO DEL HUILA
}

\author{
Marta Cecilia Vinasco Guzmán, Nelly María Méndez, \\ Luis Herney Salazar, Gina Alexandra Ayala, \\ César Augusto Munar, Vineth Munar y \\ Carlos Alirio Samboni ${ }^{20}$
}

RESUMEN

La mora de Castilla, Rubus Glaucus Bent, es una fruta de alto consumo por su excelente sabor y cualidades alimenticias. La planta que se siembra en alturas de los 1.800 a 2.200 msnm, está difundida en todo el país y es una fuente de ingresos permanentes para los productores. El género Rubus es uno de los más diversos del reino vegetal, lo que hace a la mora rica genéticamente. La planta de mora de Castilla presenta gran cantidad de espinas, de ahí la dificultad de su manejo para los procesos de recolección. En los años 90 se descubrió en la zona cafetera una variedad denominada sin espinas, que ha sido propagada ampliamente en proyectos financiados con capital oficial y privado. En la zona sur del Huila no se han evaluado aspectos relacionados con su capacidad para atender los requerimientos de la agroindustria.

Palabras clave: mora, mora sin espinas, agroindustria, NTC 4106, calidad.

\begin{abstract}
The blackberry, Rubus Glaucus Bent, is a high consumed fruit for its excellent flavor and nutritional qualities. The plant is grown at altitudes of 1800 to 2200 meters. It is spread in the whole country and is a source of permanent income for the producers. The genus Rubus is one of the most diverse plant kingdoms, making the genetically rich blackberry. The blackberry plant has lots of thorns, making it difficult to handle it in the harvest process. A new variety of blackberry was discovered in the 90's, which was called the thorn less blackberry. It has been propagated extensively in projects funded by official and private capital. The possibilities of income and production for agrobusiness of this product have not been evaluated in the southern Huila.
\end{abstract}

Key words: Blackberry, spineless blackberry, Huila, agribusiness, NTC 4106, quality.

20 Grupo de Investigación: Inyumacizo, Código del registro del grupo en Colciencias: COL0043529, Nombre del Semilero: Rosiyé, Línea de Investigación: Desarrollo Sostenible, Red de Investigación: Desarrollo Sostenible, UNAD. 


\section{Resultados}

Los datos se recogieron en los meses de agosto de 2009 a marzo de 2010, en 6 tomas de muestras de 6 cultivos en los municipios de Pitalito y San Agustín. En el tiempo de recolección, para las últimas 4 muestras se presentó el Fenómeno del Niño. Al realizar los análisis se encontró que tanto la mora con espinas como la sin espinas no presentan las variables de calidad requeridas por la agroindustria y que entre las dos variedades no se presentan diferencias significativas en ninguno de los parámetros evaluados, que fueron calidad, $\mathrm{pH}$, calibre, peso, grados Brix y sanidad, referenciados con la norma técnica de calidad de la mora NTC 4106.

\section{INTRODUCCIÓN}

En Colombia, una fruta de alto consumo es la especie Rubus Glaucus Bent, conocida como mora de Castilla, perteneciente al género Rubus, de la famila Rosaceae, uno de los más diversos del reino vegetal. La mora tiene gran aceptación para el consumo en fresco por ser rica en minerales y vitaminas y para ser procesada por su exquisito sabor y la facilidad de agroindustrialización.

La mora, como una fruta de clima frío moderado, hace parte de los cultivos representativos para los agricultores de zonas frías por su capacidad de producir ingresos, es fuente de empleo rural y, además, es una alternativa agrícola rentable frente a otros cultivos.

En todo el país y en especial en el departamento del Huila, que es el segundo productor de frutas a nivel nacional, se viene promocionando la siembra de mora sin espinas, variedad encontrada en la zona cafetera en los años noventa y hoy ampliamente difundida en varias zonas del país por sus ventajas en tamaño y producción y la facilidad en la recolección.

En las universidades del Quindío y Tecnológica de Pereira se han desarrollado investigaciones en la parte agronómica y genética de la mora cultivada en la zona cafetera. Acerca de la mora sin espinas, se encuentra en la literatura que presenta mayores niveles de grados Brix y tamaño de fruto. Sin embargo, en la zona sur del Huila, no se han hecho investigaciones acerca de las casi 100 hectáreas nuevas sembradas en esta variedad. La presente propuesta evalúa cinco parámetros de calidad de mora con y sin espinas para agroindustria: $\mathrm{pH}$, calibre, peso, grados brix y sanidad. La hipótesis estuvo orientada a demostrar si había diferencias significativas entre las dos variedades de mora.

La investigación se desarrolló con la colaboración de la Asociación de Productores de Frutas del Huila Aprofrusa en los municipios de Pitalito y San Agustín, por el semillero de investigación Rosiyé, como beneficiarios de la convocatoria 2009 -1 de SIUNAD. 


\section{OBJETIVO GENERAL}

Realizar la evaluación de cinco parámetros de calidad de la mora de Castilla Rubus Glaucus Benth variedad sin espinas, en comparación con la mora con espinas, en cultivos de los municipios de Pitalito y San Agustín.

\section{OBJETIVOS ESPECÍFICOS}

Ofrecer información científica sobre la calidad de la mora producida por la Asociación de Productores de Frutas del Huila, APROFRUSA.

Determinar la calidad de la mora producida en Pitalito y San Agustín, variedad con y sin espinas, con los indicadores solicitados por el mercado agroindustrial: sanidad, color, $\mathrm{pH}$ y grados Brix.

Comparar la calidad de la mora producida en Pitalito y San Agustín, variedad con y sin espinas, con los indicadores solicitados por la NTC 4106, los cuales son: sanidad, color, grados Brix, $\mathrm{Ph}$ y calibre.

Identificar cuál de las dos variedades de mora, con espinas o sin espinas, ofrece mejores características de calidad para el mercado agroindustrial.

\section{METODOLOGÍA}

La investigación fue realizada en 6 fincas de productores de APROFRUSA, de los municipios de Pitalito y San Agustín, en los meses de agosto de 2009 a febrero de 2010.

Tabla 1. Fincas participantes en el estudio

\begin{tabular}{|c|l|l|l|l|c|}
\hline Lote N. & Municipio & \multicolumn{1}{|c|}{ Vereda } & \multicolumn{1}{|c|}{ Finca } & \multicolumn{1}{c|}{ Propietario } & $\begin{array}{c}\text { Cultivo Con espinas } \\
\text { Sin espinas }\end{array}$ \\
\hline 1 & San Agustín & Mesitas & La Paloma & Jorge Luis Muñoz & $\mathrm{X}$ \\
\hline 2 & San Agustín & La Candela & La Planada & Guillermo Meneses & $\mathrm{X}$ \\
\hline 3 & San Agustín & La Candela & La Esperanza & Roberto Agustín Agredo & $\mathrm{X}$ \\
\hline 4 & San Agustín & La Candela & La Planada & Mabel Meneses & $\mathrm{X}$ \\
\hline 5 & Pitalito & Montecristo & Esperanza & Omar Rodríguez & $\mathrm{X}$ \\
\hline 6 & Pitalito & El Cedro & La Guaca & Fidel Obando & $\mathrm{X}$ \\
\hline
\end{tabular}

Fuente: Los Autores 


\section{Tipo de estudios}

Los métodos de investigación son inductivo y deductivo. El universo: 54 fincas donde se tienen sembrados de moras con y sin espinas, se va a hacer recolección de muestras en 5 de ellas, con una frecuencia mensual durante 6 meses, recolectando la totalidad de las moras con madurez comercial en 5 matas.

\section{Métodos}

Se emplearon los métodos inductivo y deductivo, con apoyo en métodos estadísticos que nos permitieron la presentación e interpretación de resultados. La investigación tuvo un enfoque cuantitativo y se trabajó con cifras y datos numéricos recolectados de los sitios del desarrollo del proyecto.

\section{Análisis estadístico}

El universo estuvo determinado por cincuenta y cuatro (54) fincas con áreas sembradas con mora sin espinas y mora con espinas, ubicadas en los municipios de Pitalito y San Agustín, de las cuales se tomaron cinco (5) fincas (muestra).

Para el protocolo de recolección y análisis de muestras (Antia y Torres, 1998), se hizo una adaptación del presentado en el trabajo "El manejo post-cosecha de mora (Rubus glaucus Benth)", desarrollado dentro del Programa Naciónal del SENA de Capacitación en Manejo Post-Cosecha y Comercialización de Frutas y Hortalizas, Convenio SENA - Reino Unido.

Diseño experimental: como soporte estadístico se aplica un diseño generalizado de bloques al azar, con igual número de unidades por bloque, con cinco réplicas de cada variedad.

La información recolectada, se tabuló y sometió a un análisis de varianza (Anava) generalizado de bloques al azar para determinar la variabilidad de la mora sin espinas y la mora con espinas y determinar si existían diferencias significativas. En caso de existir éstas, se aplicaría la prueba de significancia Tukey con el fin de identificar cuál variedad tuvo mejor comportamiento de acuerdo con las hipótesis planteadas.

\section{RESULTADOS}

En estos resultados de la investigación, es de anotar que hubo dos factores que incidieron: el clima que fue extremadamente seco sobre todo en las últimas 4 tomas de muestras en los meses de febrero y marzo de 2010, donde se presentó el Fenómeno del Niño y los bajos precios de la mora, que hacen que los agricultores dejen de realizar prácticas culturales o de aplicar productos orgánicos o químicos a sus cultivos. 


\section{Análisis de sanidad}

La sanidad es una variable muy importante en la agroindustria y dentro de los requisitos que debe presentar la fruta se determina que ésta debe estar libre de hongos, indicios de fermentación o sobre-maduración.

\section{Porcentaje de sanidad}

La siguiente tabla corresponde a lo encontrado, en frutos de moras con y sin espinas, sanos y enfermos.

Tabla 2. Porcentaje de sanidad de la mora producida por los dos municipios

\begin{tabular}{|l|c|c|c|}
\hline \multicolumn{1}{|c|}{ Sanidad } & Frutos Sanos & Frutos Enfermos & Total \\
\hline Pitalito & $73 \%$ & $27 \%$ & $100 \%$ \\
\hline San Agustín & $56 \%$ & $44 \%$ & $100 \%$ \\
\hline
\end{tabular}

Fuente: los autores

La grafica resultante, fue la siguiente:

Figura 1. Porcentaje de sanidad de la mora producida por los dos municipios

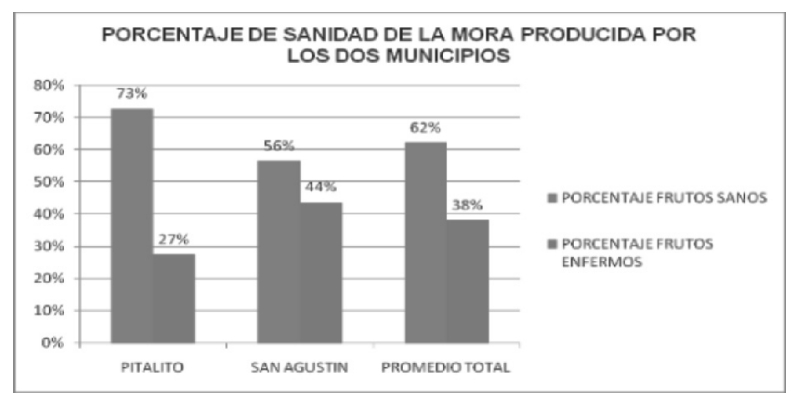

Fuente los autores

Se analizaron 9.274 frutos entre sanos y enfermos, de los cuales el $62 \%$ corresponde a frutos sanos y $38 \%$ a enfermos. El mayor porcentaje de frutos sanos corresponde a Pitalito con un $73 \%$ y le sigue San Agustín con un 56\%.

\section{Promedio frutos sanos y enfermos}

En la siguiente tabla se muestran los porcentajes de sanidad encontrados en mora con y sin espinas: 
Tabla 3. Porcentaje de frutos sanos y enfermos de la mora con y sin espinas

\begin{tabular}{|l|c|c|}
\hline \multicolumn{1}{|c|}{ Porcentaje } & Con espinas & Sin espinas \\
\hline Cantidad frutos sanos & $28 \%$ & $34 \%$ \\
\hline Cantidad frutos enfermos & $19 \%$ & $19 \%$ \\
\hline
\end{tabular}

Fuente los autores

Del total de los frutos de mora recogidos, el 62\% fueron moras sanas y el 38\% enfermas; el porcentaje de moras enfermas fue igual para las dos variedades, del 19\%.

\section{Análisis de calidad}

La calidad para la mora está definida en la norma NTC 4106 como extra (libre de todo defecto), primera y segunda.

\section{Porcentaje de calidad}

Los datos encontrados para calidad en la mora con y sin espinas, fueron los siguientes:

Tabla 4. Porcentaje de calidad en N. ${ }^{\circ}$ de frutos para las dos variables

\begin{tabular}{|l|c|c|}
\hline \multicolumn{1}{|c|}{ Calidad } & \% Frutos sin espinas & \% Frutos con espinas \\
\hline Extra & $5 \%$ & $6 \%$ \\
\hline Primera & $10 \%$ & $14 \%$ \\
\hline Segunda & $41 \%$ & $25 \%$ \\
\hline
\end{tabular}

Fuente los autores

La gráfica resultante, fue la siguiente:

Figura 2. Calidad en N. ${ }^{\circ}$ de frutos para las dos variedades

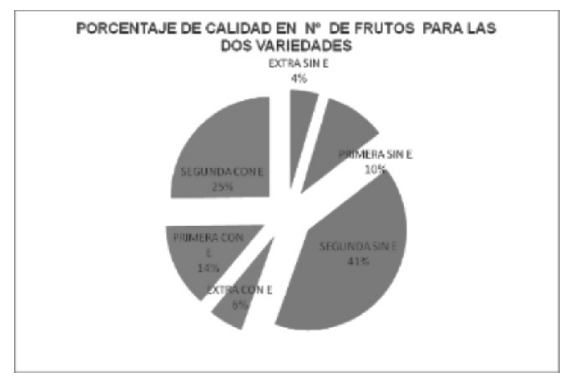

Fuente: los autores 
En la variable calidad, el 10\% del número de frutos corresponde a frutos extra, el $24 \%$ a primera y el $66 \%$ a segunda.

Del total, el $56 \%$ de los frutos fueron sin espinas, de esos el $5 \%$ es extra, el $10 \%$ es de primera y el $41 \%$ de segunda; del $44 \%$ de los frutos con espinas, el $5 \%$ fue de extra, el $14 \%$ de primera y el $25 \%$ de segunda.

\section{Análisis de calibre}

El calibre está definido en la NTC 4106, de acuerdo al tamaño de la circunferencia del fruto, como A, B, C y D en orden de tamaño.

\section{Porcentaje de calibre}

Los datos encontrados fueron:

Tabla 5. Porcentaje del calibre de los frutos con y sin espinas

\begin{tabular}{|l|l|l|}
\hline \multicolumn{1}{|c|}{ Calibre } & $\mathbf{N}^{\circ}$ de frutos & Porcentaje de frutos \\
\hline A sin espinas & 28 & $0,5 \%$ \\
\hline A con espinas & 21 & $0,4 \%$ \\
\hline B sin espinas & 300 & $5,2 \%$ \\
\hline B con espinas & 380 & $6,6 \%$ \\
\hline C sin espinas & 1392 & $24,2 \%$ \\
\hline C con espinas & 1417 & $24,6 \%$ \\
\hline D sin espinas & 1476 & $25,6 \%$ \\
\hline D con espinas & 743 & $12,9 \%$ \\
\hline Total & 5757 & \\
\hline
\end{tabular}

Fuente los autores

La gráfica resultante nos muestra de una manera más directa, lo encontrado:

De los datos obtenidos el 9\% de los frutos es de calibre A, el $11.8 \%$ es de calibre B, el $48.8 \%$ del calibre $\mathrm{C}$ y el $38.5 \%$ del calibre D.

En la variedad sin espinas, el calibre A es de $0.5 \%$, del B es de $5.2 \%$, el C de $24.2 \%$ y el D con un porcentaje de $25.6 \%$. De los frutos con espinas el calibre A con un porcentaje de $0.4 \%$, del B es de $6.6 \%$, el C de $24.6 \%$ y el D $12.9 \%$. 


\section{Análisis de pH}

El pH es una variable importante para la agroindustria porque nos indica el grado de acidez que tenga la mora, lo que incide en el sabor.

Tabla 6. Comparación de porcentajes de $\mathrm{pH}$ de mora con y sin espinas

\begin{tabular}{|l|c|c|c|c|c|}
\hline \multicolumn{1}{|c|}{ Color } & Sin espinas & Con espinas & Norma pH & \% sin espinas & \% con espinas \\
\hline Color 3 & 2,62 & 2,48 & 3,4 & $-22,9 \%$ & $-27,1 \%$ \\
\hline Color 4 & 2,64 & 2,595 & 3,1 & $-14,8 \%$ & $-16,3 \%$ \\
\hline Color 5 & 2,705 & 2,625 & 2,8 & $-3,4 \%$ & $-6,2 \%$ \\
\hline Color 6 & 2,92 & 2,815 & 2,5 & $16,8 \%$ & $12,6 \%$ \\
\hline Promedio & 2,72 & 2,63 & 2,95 & & \\
\hline
\end{tabular}

Fuente: Los autores

Analizando el $\mathrm{pH}$, el promedio de la variedad sin espinas es de 2,72 y la variedad con espinas de 2,63; y el pH promedio según la NTC 4106 debe ser de 2,95. Notándose que ninguna variedad cumple con lo exigido por la NTC 4106.

\section{Análisis grados Brix}

Los grados Brix nos indican el porcentaje de azúcar en la mora.

\section{Promedio de grados Brix}

Los datos encontrados para la totalidad de la toma de muestras:

Tabla 7. Promedio Brix - color para los dos municipios

\begin{tabular}{|l|l|l|}
\hline Color & Con o sin espinas & Promedio Brix \\
\hline 3 & Con espinas & 4,295 \\
\hline 3 & Sin espinas & 4,555 \\
\hline 4 & Con espinas & 5,45 \\
\hline 4 & Sin espinas & 5,525 \\
\hline 5 & Con espinas & 6,415 \\
\hline 5 & Sin espinas & 6,435 \\
\hline 6 & Con espinas & 7,635 \\
\hline 6 & Sin espinas & 7,58 \\
\hline 3 & NTC 4106 & 6,3 \\
\hline 4 & NTC 4106 & 6,7 \\
\hline 5 & NTC 4106 & 7,2 \\
\hline 6 & NTC 4106 & 7,7 \\
\hline
\end{tabular}

Fuente: los autores 
De los datos obtenidos en grados Brix, el color 3 en la variedad con espinas es de 4,295, el color 4 de 5,45; el color 5 de 6,415 y el color 6 con un promedio de 7,635.

En la variedad sin espinas, los grados Brix en el color 3 es de 4,555, en el color 4 de 5,525, el color 5 de 6,435, y para el color 6 un promedio de 7,58.

Las dos variedades de mora con y sin presentan los valores de Grados Brix en los colores (3, $4,5,6)$ por debajo de los establecidos en la NTC 4106, así, para la variedad con espinas el incumplimiento es el siguiente $(-32 \%,-19 \%,-11 \%,-1 \%)$; y para la variedad sin espinas es $(-28 \%,-18 \%,-11 \%,-2 \%)$.

\section{DISCUSIÓN}

Contrario a los datos encontrados en la literatura, la presente investigación muestra que las diferencias entre la mora con y sin espinas son insignificantes y que las dos variedades en cuanto a las variables analizadas son iguales.

Los datos recolectados permiten hacer otro tipo de investigaciones en la parte agronómica, pues se cuenta con datos por fecha de recolección, lote, variedad y mata y se tienen registros de seguimiento de las labores realizadas en cada cultivo.

\section{CONCLUSIONES}

Todas las variables estudiadas para la mora con y sin espinas producida en los municipios de Pitalito y San Agustín, presentan valores por debajo de la norma NTC 4106 y los grados Brix están por debajo de los exigidos por la agroindustria de 7.0.

De los análisis de varianza realizados para comparar las dos variedades de mora con y sin espinas, en todas las variables estudiadas, se encontró que las F calculadas son menores que el valor crítico, lo cual indica que las dos muestras son iguales.

No se empleó la prueba de Tukey, debido a que se encontró que las muestras son iguales y no entraría al caso realizar una comparación.

Validando la hipótesis se concluye que las dos variedades de mora con y sin espinas no presentan diferencias significativas entre las medidas de sanidad, pH, Grados Brix, calibre y peso.

\section{REFERENCIAS BIBLIOGRÁFICAS}

ANTIA, Germán Alonso y TORRES, Juan Fernando (1998), El manejo post-cosecha de mora (Rubus glaucus Benth). Serie de paquetes de capacitación sobre manejo en post-cosecha de frutas y hortalizas vols. Armenia, Quindío: Centro Agroindustrial del SENA, 1998. 
APROFRUSA. (2006), Guía de Cosecha y Poscosecha de Mora, Lulo, Granadilla y Tomate de Árbol. Pitalito: Indugráficas.

UNIVERSIDAD DEL QUINDÍO. (2007), «Memorias del Seminario de Mora.» Armenia, Quindío, 23 de Agosto. 